\title{
Impact of prolonged overfeeding on skeletal muscle mitochondria in healthy individuals
}

\author{
Frederico G. S. Toledo ${ }^{1}$ - Darcy L. Johannsen ${ }^{2}$ Jeffrey D. Covington ${ }^{2}$ • \\ Sudip Bajpeyi $^{2,3} \cdot$ Bret Goodpaster $^{4}$ - Kevin E. Conley ${ }^{5}$ - Eric Ravussin ${ }^{2}$
}

Received: 24 May 2017 / Accepted: 17 October 2017 /Published online: 17 November 2017

(C) Springer-Verlag GmbH Germany, part of Springer Nature 2017

\begin{abstract}
Aims/hypotheses Reduced mitochondrial capacity in skeletal muscle has been observed in obesity and type 2 diabetes. In humans, the aetiology of this abnormality is not well understood but the possibility that it is secondary to the stress of nutrient overload has been suggested. To test this hypothesis, we examined whether sustained overfeeding decreases skeletal muscle mitochondrial content or impairs function.

Methods Twenty-six healthy volunteers (21 men, 5 women, age $25.3 \pm 4.5$ years, BMI $25.5 \pm 2.4 \mathrm{~kg} / \mathrm{m}^{2}$ ) underwent a supervised protocol consisting of 8 weeks of high-fat overfeeding ( $40 \%$ over baseline energy requirements). Before and after overfeeding, we measured systemic fuel oxidation by indirect calorimetry and performed skeletal muscle biopsies to measure mitochondrial gene expression, content and function in vitro. Mitochondrial function in vivo was measured by ${ }^{31} \mathrm{P}$ NMR spectroscopy.

Results With overfeeding, volunteers gained $7.7 \pm 1.8 \mathrm{~kg}(\%$ change $9.8 \pm 2.3$ ). Overfeeding increased fasting NEFA, LDLcholesterol and insulin concentrations. Indirect calorimetry showed a shift towards greater reliance on lipid oxidation. In
\end{abstract}

Frederico G. S. Toledo

toledofs@upmc.edu

1 Division of Endocrinology and Metabolism, Department of Medicine, University of Pittsburgh School of Medicine, 200 Lothrop Street, BST W1054, Pittsburgh, PA 15261, USA

2 Pennington Biomedical Research Center, Baton Rouge, LA, USA

3 Department of Kinesiology, University of Texas El Paso, El Paso, TX, USA

4 Translational Research Institute for Metabolism and Diabetes, Orlando, FL, USA

5 University of Washington Medical Center, Seattle, WA, USA skeletal muscle tissue, overfeeding increased ceramide content, lipid droplet content and perilipin-2 mRNA expression. Phosphorylation of AMP-activated protein kinase was decreased. Overfeeding increased mRNA expression of certain genes coding for mitochondrial proteins $(C S, O G D H, C P T 1 B$, $U C P 3, A N T 1$ ). Despite the stress of nutrient overload, mitochondrial content and mitochondrial respiration in muscle did not change after overfeeding. Similarly, overfeeding had no effect on either the emission of reactive oxygen species or on mitochondrial function in vivo.

Conclusions/interpretation Skeletal muscle mitochondria are significantly resilient to nutrient overload. The lower skeletal muscle mitochondrial oxidative capacity in human obesity is likely to be caused by reasons other than nutrient overload per se. Trial registration ClinicalTrials.gov NCT01672632.

Keywords Human · Insulin resistance · Insulin sensitivity · Mitochondria $\cdot$ Obesity

$\begin{array}{ll}\text { Abbreviations } \\ \text { AMPK } & \text { AMP-activated protein kinase } \\ \text { ATP }_{\text {max }} & \text { Mitochondrial capacity } \\ \text { FCCP } & \begin{array}{l}\text { Carbonyl cyanide- } \\ \text { 4-(trifluoromethoxy)phenylhydrazone }\end{array} \\ \text { PAL } & \text { Physical activity level } \\ \text { PCr } & \text { Phosphocreatine } \\ \text { PGC- } & \text { Peroxisome proliferator-activated receptor- } \gamma \text { coac- } \\ 1 \alpha & \text { tivator- } 1 \alpha \\ \text { qPCR } & \text { Quantitative PCR } \\ \text { ROS } & \text { Reactive oxygen species } \\ \text { SMR } & \text { Sleep metabolic rate } \\ \text { TDEE } & \text { Total daily energy expenditure }\end{array}$




\section{Introduction}

Mitochondrial content and function are key determinants of the overall oxidative capacity and metabolic fitness of skeletal muscle tissue. Given the role of skeletal muscle in glucose metabolism, it is important to understand how dietary perturbations affect mitochondrial capacity, especially in the context of diseases such as obesity and type 2 diabetes. Lower oxidative capacity has been reported in obese individuals and in those with type 2 diabetes [1,2]. The physiological implications remain a subject of ongoing debate $[3,4]$ but it has been suggested that impaired mitochondrial capacity leads to increased deposition of ectopic lipids in skeletal muscle tissue and liver and the development of insulin resistance [1, 5-7].

A related but equally important question is why some obese individuals have a reduction in skeletal muscle oxidative capacity. The underlying pathophysiological cause(s) remain unknown. One possibility is that genetic factors may play a role. Lower mitochondrial capacity has been reported in the lean offspring of individuals with type 2 diabetes [6] and in lean African-American women at increased risk for obesity and diabetes [8]. However, some studies suggest that mitochondrial abnormalities can be acquired: short-term exposure to lipid excess was found to reduce mitochondrial capacity [9, 10]. Thus, nutrient excess may be in part responsible for the decreased mitochondrial oxidative capacity observed in obese adults and potentially in individuals with type 2 diabetes. Nevertheless, there remains a substantial gap in knowledge on how skeletal muscle mitochondria adapt to sustained nutrient overload. In contrast to findings in humans, obesity in rodents is accompanied by increased mitochondrial oxidative capacity [11-13]. Therefore, well-controlled studies in humans are essential for understanding the impact of prolonged nutrient stress on mitochondrial biogenesis and oxidative capacity in skeletal muscle.

In the present study, our goal was to determine whether sustained exposure to nutrient overload would decrease mitochondrial biogenesis, content or function. To answer this question, we overfed healthy volunteers a high-fat diet $40 \%$ above baseline energy requirements for 8 weeks under strict supervision. To examine the impact of overfeeding, we employed a range of methodologies to comprehensively measure mitochondrial biogenesis at the transcriptional level and at the whole organelle level (quantification of mitochondrial content by electron microscopy) and studied mitochondrial function in vitro (respiration and reactive oxygen species [ROS] emission) and in vivo ( ${ }^{31} \mathrm{P}$ NMR spectroscopy).

\section{Methods}

Participants The current investigation was part of a larger study on the impact of overfeeding on adipocyte characteristics and metabolic outcomes (ClinicalTrials.gov registration no. NCT01672632) [14]. We carried out a subset analysis of 26 participants ( 21 men, 5 women) in whom mitochondrial measurements were performed. The study was approved by the Pennington Biomedical Research Center Institutional Review Board. All participants provided written informed consent. Healthy, weight-stable adults aged 20-40 years, with a BMI of $22.0-32.0 \mathrm{~kg} / \mathrm{m}^{2}$, were recruited from the Baton Rouge, Louisiana community by way of advertisements. Participants had to be willing to take part in an 8 week study that resulted in a $5-10 \%$ weight gain, consume all meals at the research centre and not change their usual level of physical activity over the course of the study. Exclusion criteria included chronic use of any medication or supplement, any chronic diseases (e.g. diabetes, hypertension, heart, liver or gastrointestinal disorders), prior weight changes $(>2.5 \mathrm{~kg}$ gain or loss over the past 6 months or prior obesity), past history of eating disorders, pregnancy or breast feeding, presence of metal implants and use of illicit drugs or tobacco products. Study measurements were obtained at baseline and after overfeeding.

Overfeeding protocol Two weeks before overfeeding, baseline energy requirements were determined by measures of 14 day energy expenditure by doubly labelled water and 7 days of feeding to energy balance as previously described [15]. The baseline energy requirement was calculated as the mean of the two values and was multiplied by 1.4 to determine the overfeeding energy prescription. All meals were prepared by our metabolism kitchen using a validated 5 day rotating menu [16] composed of $41 \%$ carbohydrate, $15 \%$ protein and $44 \%$ fat (40\% saturated) [14]. Volunteers consumed all meals (three meals per day, 7 days per week for 8 weeks) at the research centre under direct supervision but were free-living for the remainder of the time. During the final days of the study (days 57-59), prior to metabolic testing, participants were again fed to energy balance using the same baseline equation but inputting their new body weight. Body fat and lean gains were determined by dual-energy x-ray absorptiometry (DEXA). The RQ was calculated from oxygen consumption $\left(\dot{V} \mathrm{O}_{2}\right)$, carbon dioxide production $\left(\dot{V}^{-O_{2}}\right)$ and urinary nitrogen measurements collected during a $23 \mathrm{~h}$ stay in a whole-room calorimeter as previously described [17]. RQ was sampled at $15 \mathrm{~min}$ intervals over $23 \mathrm{~h}$ and averaged to obtain the $24 \mathrm{~h}$ RQ. During the $24 \mathrm{~h}$, participants were provided with three meals and one snack while in the chamber, which they consumed in entirety. Sleeping metabolic rate (SMR) was calculated between 02:00 hours and 05:00 hours when infrared motion sensors were reading near-zero motion. Fasting RQ was measured the morning after sleep. Physical activity level (PAL) was calculated using estimates of total daily energy expenditure (TDEE) by doubly labelled water and SMR 
during the overnight stay in the chamber $(\mathrm{PAL}=\mathrm{TDEE} / \mathrm{SMR})$ and steps per day were measured using a validated activity monitor [18].

Muscle biopsy Vastus lateralis muscle samples were obtained in the morning by percutaneous biopsy using the Bergstrom technique [19]. After dissection, a portion of the sample was promptly frozen in liquid nitrogen for storage at $-80^{\circ} \mathrm{C}$ until biochemical analysis. A small portion was saved for electron microscopy studies after fixation and mitochondrial functional assays in fresh tissue while the remainder was processed for other in vitro assays.

Ceramide measurement Frozen muscle tissue $(50 \mathrm{mg})$ was homogenised in deionised water and protein was determined by Bio-Rad protein assay kit (Bio-Rad Laboratories, Hercules, CA, USA). After addition of an extraction standard (C17:0), each sample was subjected to a double extraction for lipids using the Folch extraction. Liquid chromatographyelectrospray ionisation tandem mass spectrometry (LC-ESI$\mathrm{MS} / \mathrm{MS}$ ) was used to measure intracellular levels of ceramides on a Waters Aquity Xevo MS/MS with ion source electrospray ionisation operated in the positive mode. According to the retention times of standards, common product ion and ions reflecting fatty acid substituents, all target ceramides were quantified as previously described [20].

Gene expression and protein content Total RNA was extracted using a miRNeasy kit (Qiagen, Germantown, MD, USA) and the yield determined by spectrophotometry (NanoDrop Technologies, Wilmington, DE, USA). From each sample of muscle tissue, $200 \mathrm{ng}$ was reverse transcribed to cDNA by reverse transcription (Applied Biosystems kit). Real-time quantitative PCR (qPCR) was performed with an API PRISM 7900 (Applied Biosystems, Foster City, CA, USA) and TaqMan gene expression assays for selected genes: adenine nucleotide translocator ( $A N T 1)$, citrate synthase $(C S)$, oxoglutarate dehydrogenase $(O G D H)$, cytochrome c oxidase $(C O X)$, uncoupling protein 3 (UCP3), carnitine palmitoyltransferase $1(C P T 1 B)$ and perilipin-2 (ADRP [also known as PLIN2]) and NF-kB p65 (RELA). Samples were run in duplicate and normalised for $R P L P O$ as an internal control. Relative mRNA expression was calculated as fold change from baseline.

Western blotting was employed to measure the total protein levels of AMP-activated protein kinase (AMPK) $\alpha$ and phospho-AMPK $\alpha$ (Thr172). The ratio between the two was used to determine the degree of AMPK phosphorylation. Cell Signaling Technologies (Danvers, MA, USA) provided the primary antibodies (AMPK $\alpha$ no. 2532; phospho-AMPK $\alpha$ no. 2531). Glyceraldehyde 3-phosphate dehydrogenase (GAPDH) was used as an internal control (no. AB9484; AbCam, Toronto, ON, Canada). Total protein was collected using RIPA buffer supplemented with $2 \%$ (vol./vol.) protease inhibitor cocktail, $2 \%$ (vol./vol.) phosphatase inhibitor cocktail 2 and 2\% (vol./vol.) phosphatase inhibitor cocktail 3 (Sigma, St Louis, MO, USA). Bands were visualised and quantified using an Odyssey 9120 infrared imaging system (Li-Cor, Lincoln, NE, USA).

Mitochondrial content and transmission electron microscopy studies A portion of skeletal muscle from a subset of participants was taken for systematic quantification of mitochondrial content by transmission electron microscopy as previously described [21, 22]. For each sample, 18-20 random, independent, longitudinal axis micrographs of intermyofibrillar fields were taken at 36,600× magnification (JEM-1210; Jeol, Tokyo, Japan). Samples were de-identified and analysis was blinded. Mitochondrial content was expressed as mitochondrial volume density (the cell fraction occupied by mitochondria according to stereological principles) and measured by the point-sampling technique of classical stereology assisted by specialised digital imaging software (Metamorph 6.3, Molecular Devices, Sunnyvale, CA). Grid density consisted of 144 symmetrically spaced intersection points. Lipid droplet content was also systematically measured on the same micrographs using a grid density of 400 intersection points per image and expressed as lipid droplet volume density. In addition to transmission electron microscopy measurements, mitochondrial content was determined by succinate dehydrogenase histochemical staining [23]. Mitochondrial DNA abundance was determined by the ratio of mtDNA to nuclear DNA copy numbers using qPCR as described [24].

Mitochondrial extraction Immediately following tissue collection, 100-150 mg of whole muscle was minced in $1 \mathrm{ml}$ icecold mitochondrial extraction buffer (composition in $\mathrm{mmol} / \mathrm{l}$ : $5 \mathrm{MgCl}_{2}, 100 \mathrm{KCl}, 40$ Tris-HCl, 10 Tris-base, 1 EDTA, 1 ATP; $\mathrm{pH}$ 7.5) and subjected to a protease digestion (Type XXIV; Sigma) for 7 min [25], which was terminated by adding $1 \mathrm{ml}$ of extraction buffer. Tissue was homogenised and centrifuged at $700 \mathrm{~g}$ and the supernatant fraction was collected and centrifuged again at $14,000 \mathrm{~g}$ to collect a mitochondria-enriched pellet. After two re-suspensions and centrifugations at $7000 \mathrm{~g}$ and $3500 \mathrm{~g}$, the final pellet was resuspended in ice-cold mannitol-sucrose solution (composition in mmol/l: 220 mannitol, 70 sucrose, 10 Tris-HCl, 1 EGTA; $\mathrm{pH}$ 7.4). Protein concentration was determined by spectrophotometry using the bicinchoninic acid method (Pierce BCA Protein Assay Kit; Thermo Fisher Scientific, Rockford, IL, USA).

Mitochondrial respiration Oxygen consumption was measured in isolated mitochondria using a Clark-type oxygen electrode (Hansatech Instruments, Norfolk, UK) surrounded 
by a temperature-controlled water-jacketed glass chamber to maintain the temperature at $37^{\circ} \mathrm{C}$. Mitochondria were suspended at a concentration of $80 \mu \mathrm{g} / 400 \mu \mathrm{l}$ in Wander's Respiration Buffer and the measurement was carried out in a medium containing (in mmol/l) $100 \mathrm{KCl}, 50 \mathrm{MOPSO}, 10$ $\mathrm{K}_{2} \mathrm{HPO}_{4}, 10 \mathrm{MgCl}_{2}, 1$ EDTA, 20 glucose, 5 glutamate and $0.2 \%$ (wt/vol.) BSA. Mitochondrial respiration was measured in the presence of glutamate/malate/pyruvate after addition of sodium pyruvate $(1.25 \mathrm{mmol} / \mathrm{l})$ and malate $(1.25 \mathrm{mmol} / \mathrm{l})$. After reaching a stable rate, coupled respiration was initiated by adding ADP $(0.375 \mathrm{mmol} / \mathrm{l})$. After determination of the maximal ADP-stimulated respiration, oligomycin $(1.6 \mu \mathrm{g} / \mathrm{ml})$ was added to block complex V (ATP synthase) and provide a measure of non-ADP coupled respiration (i.e. leak-mediated). The respiratory control ratio was calculated as the rate of ADPstimulated respiration divided by leak-mediated respiration. Carbonyl cyanide-4-(trifluoromethoxy)phenylhydrazone (FCCP) $(3 \mu \mathrm{mol} / \mathrm{l})$ was added to measure maximal uncoupled mitochondrial respiration.

ROS emission Mitochondrial ROS production was measured as the $\mathrm{H}_{2} \mathrm{O}_{2}$ emission rate from methods adapted from Seifert et al [26] and St-Pierre et al [27], employing complex III inhibition to enhance mitochondrial $\mathrm{H}_{2} \mathrm{O}_{2}$ production. Briefly, mitochondria extracted from $200 \mathrm{mg}$ of muscle tissue (using the methods of Chappell and Perry [28, 29]) were suspended in a reaction medium containing $120 \mathrm{mmol} / \mathrm{l}$ $\mathrm{KCl}, 5 \mathrm{mmol} / 1 \mathrm{KH}_{2} \mathrm{PO}_{4}, 1 \mathrm{mmol} / \mathrm{l} \mathrm{EGTA}, 5 \mathrm{mmol} / 1 \mathrm{MgCl}_{2}$, $3 \mathrm{mmol} / 1$ Hepes (pH 7.4), $1 \mathrm{U} / \mathrm{ml}$ superoxide dismutase, $5 \mu \mathrm{mol} / \mathrm{l}$ antimycin and $0.3 \%$ (wt/vol.) defatted BSA. The $\mathrm{H}_{2} \mathrm{O}_{2}$ emission rate was measured fluorometrically using $p$ hydroxyphenylacetate $(165 \mu \mathrm{g} / \mathrm{ml})$ and horseradish peroxidase $(9 \mathrm{U} / \mathrm{ml})$ in the medium. The reaction was started by adding substrate (either $60 \mu \mathrm{mol} / \mathrm{l}$ palmitoyl-carnitine or $10 \mathrm{mmol} / \mathrm{l}$ succinate) in the presence of $5 \mu \mathrm{mol} / 1$ rotenone. $\mathrm{H}_{2} \mathrm{O}_{2}$ emission was monitored for $25 \mathrm{~min}$ at $37^{\circ} \mathrm{C}$ at an excitation wavelength of $320 \mathrm{~nm}$ and emission wavelength of $400 \mathrm{~nm}$.

Mitochondrial function in vivo Mitochondrial capacity $\left(\mathrm{ATP}_{\max }\right)$ was measured in the vastus lateralis of the dominant leg (same site as the biopsy) using ${ }^{31} \mathrm{P}$ NMR spectroscopy as previously described [30,31]. Following the acquisition of a fully relaxed spectrum, ${ }^{31} \mathrm{P}$ spectra were acquired every $1.5 \mathrm{~s}$ during a 24,30 or $36 \mathrm{~s}$ ballistic exercise involving 'kicking' against Velcro straps positioned tightly across the leg and thigh. Ballistic contractions are used to ensure activation of all muscle fibres, so that exercise activates all muscle fibres [32]. Exercise time and intensity were targeted to drop phosphocreatine (PCr) by 33-50\% of basal levels and to avoid a $\mathrm{pH}<6.8$, as lower $\mathrm{pH}$ inhibits oxidative phosphorylation and results in artificially low values [32]. $\mathrm{ATP}_{\max }$ was calculated using the PCr recovery time constant $(\tau)$ and
$[\mathrm{PCr}]_{\text {rest }}: \mathrm{ATP}_{\max }=[\mathrm{PCr}]_{\text {rest }} / \tau .[\mathrm{PCr}]$ level was set at $27 \mathrm{mmol} / \mathrm{l}$ as found previously by direct measurement in muscle tissue by HPLC [33]. The reproducibility of ATP max $_{\text {mea- }}$ surements has been published $[34,35]$ and the $\mathrm{CV}$ is $\pm 7.0 \%$.

Statistics All statistical tests were two-tailed with an $\alpha$ set at 0.05 . Continuous variables were pre-examined for normal distribution by the Shapiro-Wilk test. Changes after overfeeding were analysed by paired $t$ tests. Non-normally distributed variables (mRNA expression) were analysed by the Wilcoxon signed rank test. The main endpoints of interest were changes in mitochondria-related gene expression, mitochondrial content and mitochondrial function. Multivariate regression analysis was employed to test the effect of time vs weight gain on the change in TDEE. Means \pm SD are reported unless shown otherwise.

\section{Results}

Effects of overfeeding on adiposity, substrate metabolism and systemic markers of lipid overload Table 1 shows the characteristics of the participants before and after 8 weeks of overfeeding. Body weight and adiposity (BMI and \% fat) were substantially increased by overfeeding. The mean weight gain from baseline was $7.7 \pm 1.8 \mathrm{~kg}(9.8 \pm 2.3 \%)$ and the mean gain in fat mass was $4.4 \pm 1.2 \mathrm{~kg}$. TDEE increased after overfeeding $(p<0.01)$. As assessed by multiple regression analysis, the increase in TDEE was accounted for by the increase in weight $(p<0.01)$. There were no significant changes in PAL measured by doubly labelled water (TDEE/SMR) or by activity monitors (no. of steps/day).

Weight gain was accompanied by a rise in fasting NEFA, LDL-cholesterol and insulin but not triacylglycerol levels. Overfeeding caused fuel oxidation to shift towards greater reliance on fat relative to glucose, as indicated by decreases in fasting and $24 \mathrm{~h}$ RQs $(p<0.05)$.

Changes in markers of nutrient overload in muscle As shown in Fig. 1a, after overfeeding total ceramide content in skeletal muscle was increased by more than twofold. The increase was attributed to broad changes in several ceramide subspecies, rather than a single one (Fig. 1b). In addition, intramyocellular lipid droplet content was markedly increased after overfeeding (Fig. 1c). We then examined whether there were coordinated cellular responses to fuel excess. After overfeeding, there was a significant decrease in the ratio of phosphorylated AMPK to total AMPK (Fig. 2a-c). Using qPCR, we found that the mRNA expression of RELA, which codes for $\mathrm{p} 65 / \mathrm{NF}-\mathrm{KB}$, increased after overfeeding. However, the $p$ value $(p=0.06)$ was only close to the margin of statistical significance (Fig. 2d). Perilipin-2 mRNA expression increased after overfeeding 
Table 1 Participant characteristics before and after 8 weeks of overfeeding $(n=26)$

\begin{tabular}{|c|c|c|c|}
\hline Characteristic/variable & Before & After & $p$ value \\
\hline Male sex & $21(81)$ & & \\
\hline \multicolumn{4}{|l|}{ Race } \\
\hline White & $17(65)$ & & \\
\hline Black & $9(35)$ & & \\
\hline Age, years & $25.3 \pm 4.5$ & & \\
\hline Range & $20-36$ & & \\
\hline Height, $\mathrm{cm}$ & $176.4 \pm 8.5$ & & \\
\hline Weight, $\mathrm{kg}$ & $79.3 \pm 9.5$ & $87.1 \pm 10.3$ & $<0.001$ \\
\hline BMI, $\mathrm{kg} / \mathrm{m}^{2}$ & $25.5 \pm 2.4$ & $27.9 \pm 2.7$ & $<0.001$ \\
\hline Body fat, $\%$ & $22.9 \pm 6.9$ & $25.8 \pm 6.8$ & $<0.001$ \\
\hline Fat mass, $\mathrm{kg}$ & $17.9 \pm 4.8$ & $22.3 \pm 5.5$ & $<0.001$ \\
\hline TDEE, kJ & $12,665 \pm 2402$ & $13,682 \pm 2431$ & $<0.01$ \\
\hline PAL (TDEE/SMR) & $1.79 \pm 0.32$ & $1.78 \pm 0.26$ & 0.98 \\
\hline No. of steps per day & $9654 \pm 3687$ & $8938 \pm 3139$ & 0.88 \\
\hline Fasting NEFA, mmol/1 & $0.27 \pm 0.10$ & $0.32 \pm 0.12$ & $<0.05$ \\
\hline Fasting triacylglycerol, $\mathrm{mmol} / \mathrm{l}$ & $0.91 \pm 0.42$ & $1.02 \pm 0.83$ & 0.39 \\
\hline Fasting LDL-cholesterol, mmol/l & $2.56 \pm 0.54$ & $2.96 \pm 0.65$ & $<0.01$ \\
\hline Fasting glucose, $\mathrm{mmol} / \mathrm{l}$ & $5.2 \pm 0.4$ & $5.0 \pm 0.4$ & $<0.05$ \\
\hline Fasting insulin, pmol/1 & $32.6 \pm 20.8$ & $51.4 \pm 36.1$ & $<0.01$ \\
\hline Fasting RQ & $0.89 \pm 0.03$ & $0.86 \pm 0.04$ & $<0.05$ \\
\hline $24 \mathrm{~h}$ RQ & $0.90 \pm 0.02$ & $0.89 \pm 0.03$ & $<0.05$ \\
\hline
\end{tabular}

Data are expressed as $n(\%)$ or mean $\pm \mathrm{SD}$, unless stated otherwise $(p=0.03)$ (Fig. 2e), consistent with a coordinated cellular response to accommodate increased lipid storage.

Effect of overfeeding on gene expression To understand whether overfeeding elicited a coordinated genomic response that might affect mitochondrial biogenesis, we measured the mRNA expression of several genes relevant to mitochondria (Fig. 3). After overfeeding, there was a change in the mRNA expression of genes coding for sirtuin $1(p<0.05)$ and peroxisome proliferator-activated receptor- $\gamma$ coactivator- $1 \alpha$ (PGC$1 \alpha)(p=0.10)$, two important regulators of mitochondrial biogenesis. Overfeeding also increased the expression of genes coding for certain mitochondrial proteins (citrate synthase, oxoglutarate dehydrogenase, carnitine palmitoyltransferase $1 \mathrm{~B}$, uncoupling protein 3 and adenine nucleotide translocator 1). Cytochrome c oxidase mRNA expression was not changed.

Effect of overfeeding on mitochondrial content and function Although gene expression was upregulated by overfeeding, it was not accompanied by a change in mitochondrial content. Transmission electron microscopy revealed that mitochondrial content was similar before and after overfeeding (Fig. 4). To corroborate these findings, other markers of
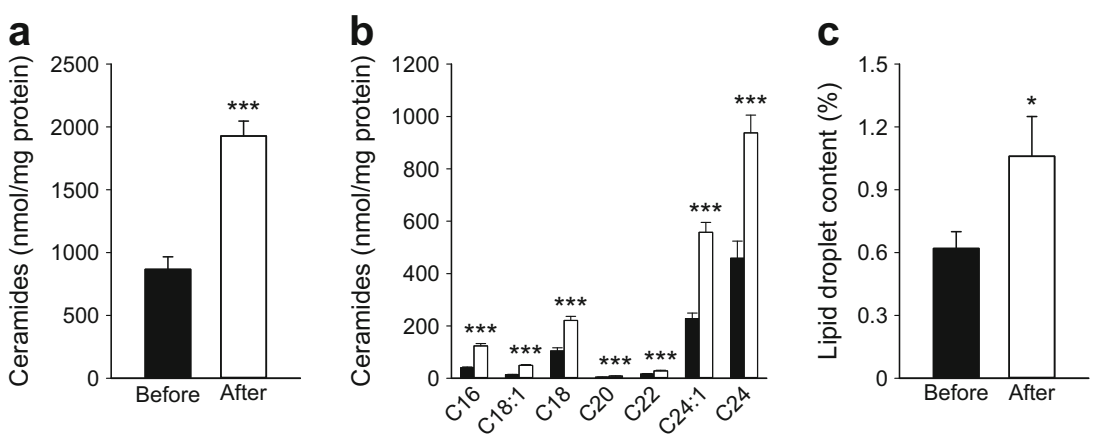

Fig. 1 Effect of overfeeding on skeletal muscle ceramides and lipid droplet content. (a, b) After overfeeding, total ceramide content in skeletal muscle was increased (a) but the change was not specific to any one ceramide subspecies (b). (c) Intramyocellular lipid droplet content was increased by overfeeding. Black bars, before overfeeding; white bars, after overfeeding. Data are means \pm SEM; $n=26$ (a, b) or 12 (c). $* p<0.05$ and $* * * p<0.001$ vs before overfeeding 

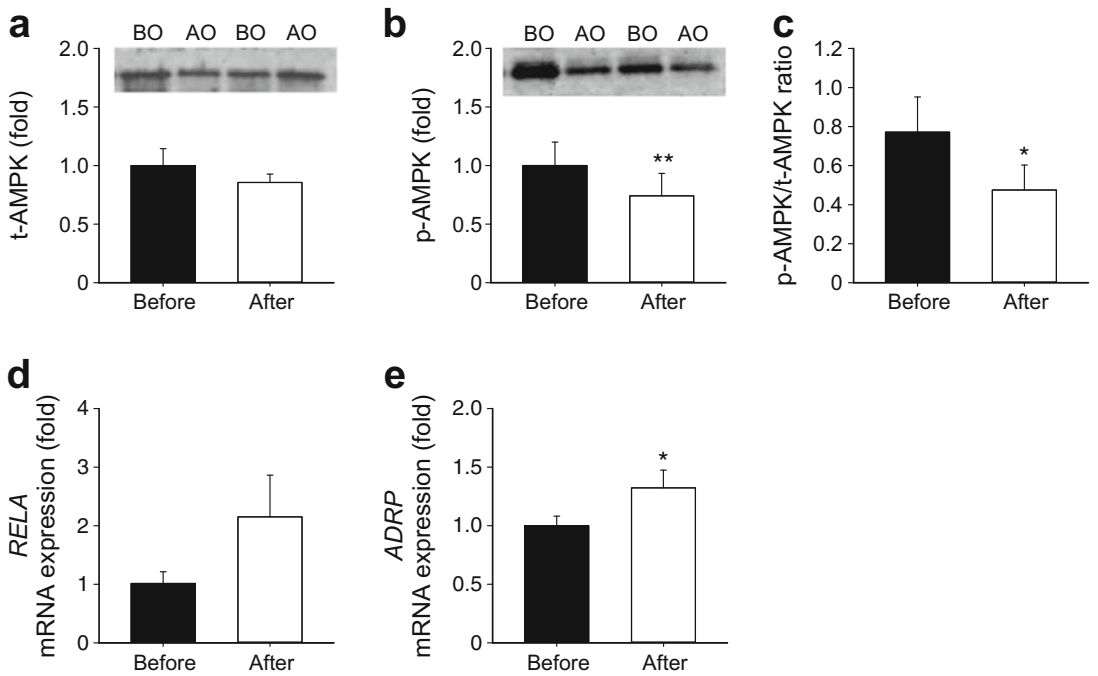

Fig. 2 Skeletal muscle cell responses to nutrient excess. After overfeeding, there were several changes indicative of cellular responses to nutrient overload. (a-c) Total AMPK (t-AMPK) protein content did not change after overfeeding (a) but phosphorylated AMPK (p-AMPK) was lower after overfeeding (b). Consequently, the p-AMPK/t-AMPK ratio was reduced after overfeeding (c). Insets show representative western blots from two participants before overfeeding (BO) and after overfeeding

mitochondrial content were examined. Succinate dehydrogenase staining did not change after overfeeding $(30.2 \pm 7.0 \mathrm{vs}$ $30.3 \pm 6.8$ (arbitrary units), $p=0.93, n=21$ ) and neither did mtDNA content $\left(11.3\right.$ vs $9.3 \times 10^{2} \mathrm{mtDNA} /$ nuclear DNA, $p=0.42, n=16$ ).

Next, we examined whether overfeeding affected mitochondrial function. There was neither a decrease nor an increase in mitochondrial respiration, whether measured in the basal leak state (non-ADP-stimulated) or ADPstimulated state (Fig. 5a). Similarly, overfeeding did not

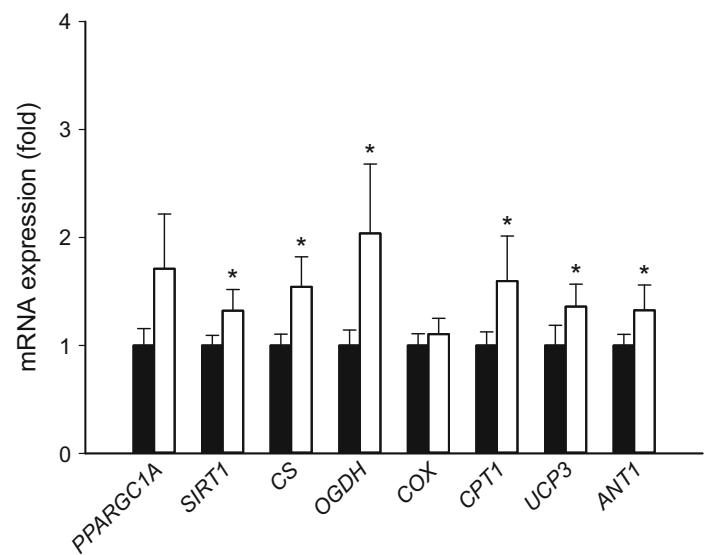

Fig. 3 Effect of overfeeding on skeletal muscle mRNA expression of mitochondria-related genes. After overfeeding, there was an increase in the expression of genes related to mitochondria. mRNA was normalised for $R P L P O$ and expressed as relative fold difference from baseline average. Black bars, before overfeeding; white bars, after overfeeding. Data are means \pm SEM; $n=24-26$. $* p<0.05$ vs before overfeeding (Wilcoxon signed rank test)
(AO). (d) After overfeeding, p65/NF- $\mathrm{kB}$ (RELA) mRNA expression increased non-significantly ( $p=0.06$; Wilcoxon test). (e) Perilipin-2 $(A D R P)$ mRNA expression significantly increased after overfeeding (Wilcoxon test). Data are means \pm SEM and expressed as relative to baseline; $n=20(\mathbf{a}-\mathbf{c}), 26(\mathbf{d})$ or $25(\mathbf{e}) . * p<0.05$ and $* * p<0.01$ vs before overfeeding

affect maximal respiration in uncoupled conditions ( $p=0.31$ ), suggesting that it had no impact on electron transport chain activity. The respiratory control ratio was unchanged (Fig. 5b). Mitochondrial ROS emission did not increase after overfeeding, whether measured with

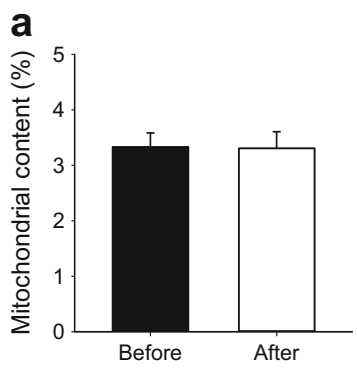

b

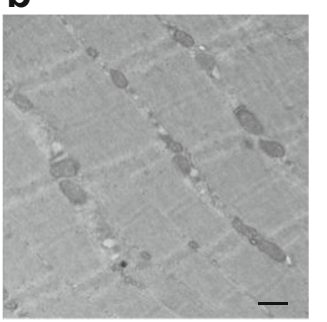

C

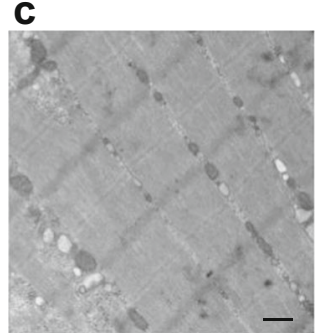

Fig. 4 Effect of overfeeding on mitochondrial content quantified by electron microscopy. (a) Transmission electron microscopy with quantification by stereology was used to directly measure mitochondrial content in skeletal muscle. There were no differences in mitochondrial content before and after overfeeding. Data are means \pm SEM $(n=12)$. (b, c) Representative micrographs of skeletal muscle before (b) and after (c) overfeeding are shown (scale bars, $500 \mathrm{~nm}$ ) 

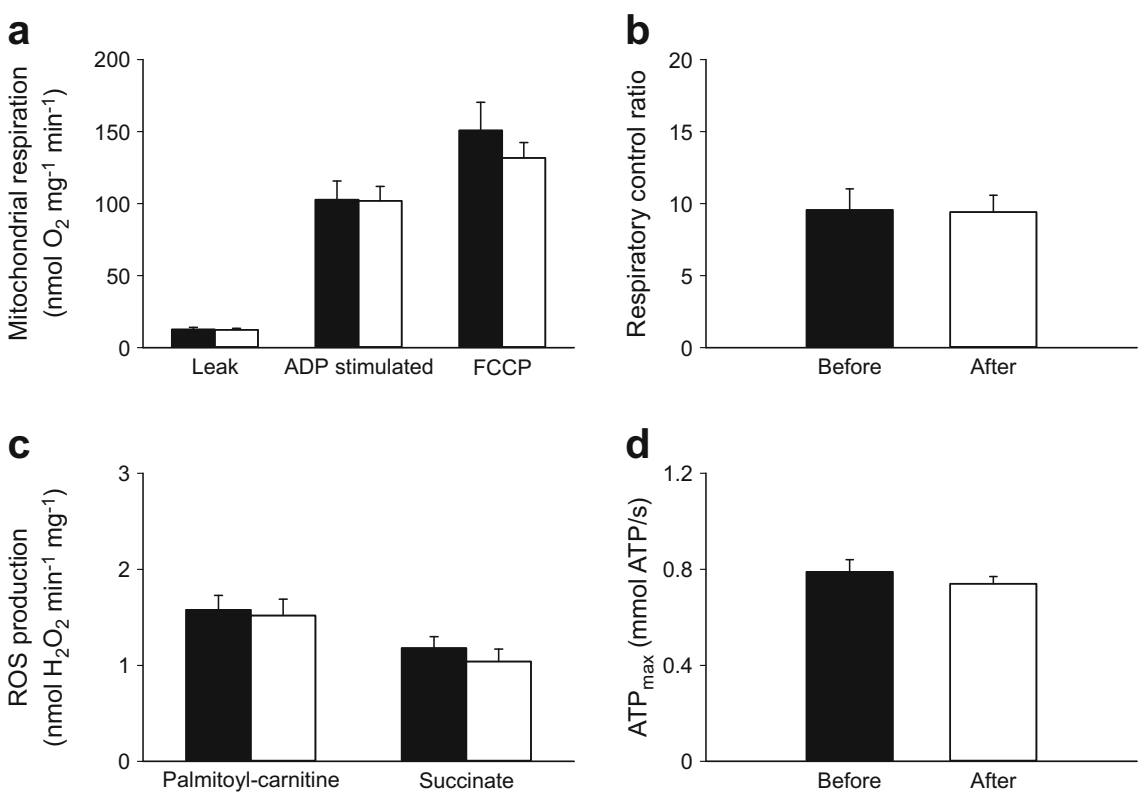

Fig. 5 Effect of overfeeding on mitochondrial function in vitro and in vivo. After overfeeding, there were no changes in mitochondrial respiration, coupling (respiratory control ratio), ROS emission or oxidative phosphorylation in vivo. (a) Mitochondrial respiration in the basal leak state, ADP-stimulated state and during maximally uncoupled conditions

palmitate or succinate as substrates (Fig. 5c). Finally, measurement of mitochondrial oxidative phosphorylation in vivo by ${ }^{31} \mathrm{P}$ NMR spectroscopy confirmed the findings observed in vitro (Fig. 5d).

\section{Discussion}

In this study, we tested whether prolonged overfeeding with a high-energy, high-fat diet would decrease mitochondrial oxidative capacity in the skeletal muscle of healthy individuals. Surprisingly, neither mitochondrial content nor function (in vitro and in vivo) was affected by overfeeding. The overfeeding protocol was successful in achieving a state of systemic lipid overload as reflected by a weight gain of $\sim 10 \%$ from baseline, a significant rise in plasma NEFA concentrations and a shift towards greater lipid oxidation. In skeletal muscle, nutrient overload was also observed. Overfeeding increased both intramyocellular lipid droplet content and ceramides (lipotoxic species that accumulate in states of fatty acid excess and adversely affect metabolism). Interestingly, some studies have implicated ceramides as being harmful to mitochondria [36, 37], although we found no such evidence in our study. Coordinated cellular responses sensing fuel excess were also noted. For instance, AMPK phosphorylation was decreased after overfeeding. AMPK activity is activated by phosphorylation during fuel deprivation and, conversely, is inhibited during fuel excess. RELA/p65 ( $\left.\mathrm{NF}_{-} \mathrm{B}\right)$ was
(FCCP). (b) Respiratory control ratio. (c) ROS emission. (d) Muscle oxidative phosphorylation in vivo measured by ${ }^{31} \mathrm{P}$ NMR spectroscopy. Black bars, before overfeeding; white bars, after overfeeding. Data are means \pm SEM; $n=21(\mathbf{a}, \mathbf{b}), 17(\mathbf{c})$ or $24(\mathbf{d})$

upregulated after overfeeding, but the $p$ value for after vs before overfeeding did not reach the threshold of statistical significance. It is hard to interpret whether the increase was simply due to random chance, although upregulation by nutrient excess is the expected biological response of this biomarker. Finally, overfeeding augmented perilipin-2 mRNA expression, suggesting a genomic response to accommodate the need for increased lipid storage. Together, the preponderance of these findings shows that a state of high-energy, high-fat overload was achieved both systemically and at the intramyocellular level and that muscle cells responded to nutrient excess. Yet, despite the challenge of nutrient excess both mitochondrial content and function were unchanged.

In studies employing an acute lipid infusion or a short-term exposure to a high-fat diet, lipid overload downregulates genes necessary for mitochondrial biogenesis $[9,10]$. Here, we found that prolonged overfeeding did not inhibit the expression of genes coding for certain mitochondrial proteins or for SIRT1 and PGC-1 $\alpha$, which are key regulators of mitochondrial biogenesis. Instead, upregulation was observed. While we did not conduct studies to determine whether the levels of each of those proteins also changed, any purported changes in those proteins did not translate into physiologically meaningful differences in mitochondrial mass; all methods to measure the latter corroborated the finding that organelle content was unchanged by overfeeding. Likewise, any hypothetical changes in those proteins did not have a meaningful 
functional impact on mitochondrial respiration or on oxidative phosphorylation. Although we did not also assess respiration with permeabilised fibres methodology, measurements of oxidative phosphorylation in vivo confirmed our findings in vitro. One limitation of our data is that subtle changes in relative fuel oxidation capacity (lipids vs carbohydrates) could have been missed without additional biochemical assessments of enzymatic activity of specific mitochondrial proteins or pathways. Similarly, cellular markers of oxidative stress and/ or damage were not measured given the limited muscle sample availability. Nevertheless, our data strongly suggest that mitochondrial content and function are remarkably resilient to the metabolic stress of prolonged nutrient overload.

Although adults with obesity or type 2 diabetes are reported to have lower oxidative capacity in skeletal muscle $[1,2,38]$, mostly due to reduced mitochondrial content [2], the aetiology remains unclear. Some studies have suggested that lipid excess decreases mitochondrial biogenesis. For instance, the expression of nuclear-encoded mitochondrial genes and PGC$1 \alpha$ in skeletal muscle is suppressed by infusion of lipid for $48 \mathrm{~h}$ [9]. In another study in healthy volunteers, 3 days of a high-fat isoenergetic diet decreased the expression of PGC- $1 \alpha$ and proteins linked to mitochondria [10]. While such studies suggested that short-term nutritional challenges suppress mitochondrial biogenesis, they still left open the question of whether sustained nutrient overload eventually lowers mitochondrial content and/or function. Moreover, studies to date have relied on surrogate markers (e.g. mRNA) of mitochondrial biogenesis, which may lead to incorrect conclusions about mitochondrial content and functional capacity. Our study addresses these questions and shows that sustained nutrient overload does not inhibit mitochondrial biogenesis, content or function.

In addition to demonstrating the resilience of mitochondria in the face of sustained nutrient overload, our study provides new insights into the biology of mitochondria in obesity. Most importantly, nutrient overload per se seems unlikely to be the sole explanation for the lower mitochondrial capacity observed in obesity and potentially also in type 2 diabetes. This may explain why weight loss often does not improve mitochondrial capacity in skeletal muscle. For instance, in obese individuals weight loss alone does not improve mitochondrial oxidative capacity despite a decrease in intramyocellular lipid content and metabolic improvements in skeletal muscle [39]. Even the extreme weight loss achieved by bariatric surgery is not sufficient to increase mitochondrial oxidative capacity in skeletal muscle $[40,41]$. Together with the present data, we conclude that nutrient excess alone is not the main determinant of the lower mitochondrial capacity observed in obesity. Therefore, the pathophysiology of this reduced mitochondrial capacity must involve other aetiologies. One potential aetiology is lipid- induced oxidative stress, although we could not test such a hypothesis because emission of ROS did not increase in our study. Since obesity is often associated with a sedentary lifestyle, another hypothesis worth considering is an interaction between nutrient overload and sedentary behaviour, in a manner that only when both are present do mitochondrial abnormalities emerge. It has been demonstrated previously that mitochondrial capacity improves in conditions of a hypoenergetic diet only when increased physical activity is present [39], pointing to physical activity as the key regulator of mitochondrial content/function. In our study, participants were not sedentary and activity levels did not decline with overfeeding, which might have protected them against the stress of nutrient excess. Studies specifically focused on the interaction between physical activity and overfeeding would help answer this question.

The strengths of our study include the robust degree of weight gain achieved, the relatively long duration of overfeeding and a very comprehensive examination of mitochondrial content and function. To our knowledge, this is the first study to investigate the effects of overfeeding for this long duration and with such remarkable weight gain. In another overfeeding study [42], participants gained about 3.7\% of their baseline weight after 28 days and no decreases in mitochondrial protein concentrations were detected. Despite the relatively modest weight gain and shorter study duration, the results are in line with our findings. In our study, however, we employed a more comprehensive approach that placed greater emphasis on direct measurements of mitochondrial content and function, rather than just measuring surrogate markers. No studies employing electron microscopy to examine the effects of overfeeding on mitochondria have been previously reported. This is relevant because surrogate markers of mitochondrial content can be misleading indicators, especially in the context of lifestyle interventions [22, 39, 43].

To our knowledge, this is the longest overfeeding study conducted to comprehensively study mitochondria. Yet, the possibility that mitochondrial capacity deteriorates only after longer periods, perhaps years, remains a consideration. This is a limitation of any overfeeding study as feeding individuals to obesity levels over long periods would be ethically unacceptable. Nonetheless, mitochondrial turnover occurs relatively rapidly-downregulation of mitochondrial capacity can be observed after just 1 week of leg immobilisation [44] or 2 weeks of bed rest [45]. Our study far exceeded these time frames and, therefore, it is unlikely that 8 weeks was insufficient to observe a decline in mitochondria. Another limitation of our findings is that only healthy young individuals were studied. It is unclear whether different results would be observed in individuals with established abnormalities in mitochondrial homeostasis, such as the elderly and individuals with 
type 2 diabetes. Studies specifically devoted to investigating those populations are warranted to answer those questions.

In summary, our data suggest that skeletal muscle mitochondrial content and function are remarkably resilient to nutrient overload. We propose that the lower mitochondrial capacity observed in obesity and type 2 diabetes is not initiated by nutrient overload per se and that either other pathophysiological factors are responsible or additional perturbations need to occur in conjunction with nutrient overload.

Acknowledgements We thank D. Stoltz (Center for Biological Imaging, University of Pittsburgh, PA, USA) for assistance with electron microscopy and E. Leachman (Dept of Medicine, University of Pittsburgh, PA, USA) and P. Coen (Translational Research Institute for Metabolism and Diabetes, USA) for assistance with succinate dehydrogenase histology.

Data availability All data generated or analysed during this study are included in this published article.

Funding This work was supported by NIH grants R01DK060412 and K01DK89005 and by NORC Center Grant P30DK72476.

Duality of interest The authors declare that there is no duality of interest associated with this manuscript.

Contribution statement FGST and ER designed the study, analysed the data and wrote the manuscript. FGST was responsible for the study concept. All authors participated in data acquisition, revised the manuscript critically for intellectual content and approved the final version. ER is the guarantor of this work.

\section{References}

1. Kelley D, He J, Menshikova E, Ritov V (2002) Dysfunction of mitochondria in human skeletal muscle in type 2 diabetes mellitus. Diabetes 51:2944-2950

2. Chomentowski P, Coen PM, Radikova Z, Goodpaster BH, Toledo FG (2011) Skeletal muscle mitochondria in insulin resistance: differences in intermyofibrillar versus subsarcolemmal subpopulations and relationship to metabolic flexibility. J Clin Endocrinol Metab 96:494-503

3. Toledo FG (2014) Mitochondrial involvement in skeletal muscle insulin resistance. Diabetes 63:59-61

4. Holloszy JO (2013) Deficiency of mitochondria in muscle does not cause insulin resistance. Diabetes 62:1036-1040

5. Kelley DE, Goodpaster B, Wing RR, Simoneau JA (1999) Skeletal muscle fatty acid metabolism in association with insulin resistance, obesity, and weight loss. Am J Phys 277:E1130-E1141

6. Petersen KF, Dufour S, Befroy D, Garcia R, Shulman GI (2004) Impaired mitochondrial activity in the insulin-resistant offspring of patients with type 2 diabetes. N Engl J Med 350:664-671

7. Goodpaster BH (2013) Mitochondrial deficiency is associated with insulin resistance. Diabetes 62:1032-1035

8. DeLany JP, Dube JJ, Standley RA et al (2014) Racial differences in peripheral insulin sensitivity and mitochondrial capacity in the absence of obesity. J Clin Endocrinol Metab 99:4307-4314
9. Richardson DK, Kashyap S, Bajaj M et al (2005) Lipid infusion decreases the expression of nuclear encoded mitochondrial genes and increases the expression of extracellular matrix genes in human skeletal muscle. J Biol Chem 280:10290-10297

10. Sparks LM, Xie H, Koza RA et al (2005) A high-fat diet coordinately downregulates genes required for mitochondrial oxidative phosphorylation in skeletal muscle. Diabetes 54:1926-1933

11. Hancock CR, Han DH, Chen M et al (2008) High-fat diets cause insulin resistance despite an increase in muscle mitochondria. Proc Natl Acad Sci U S A 105:7815-7820

12. Holloway GP, Gurd BJ, Snook LA, Lally J, Bonen A (2010) Compensatory increases in nuclear $\mathrm{PGC} 1 \alpha$ protein are primarily associated with subsarcolemmal mitochondrial adaptations in ZDF rats. Diabetes 59:819-828

13. Turner N, Bruce CR, Beale SM et al (2007) Excess lipid availability increases mitochondrial fatty acid oxidative capacity in muscle: evidence against a role for reduced fatty acid oxidation in lipidinduced insulin resistance in rodents. Diabetes 56:2085-2092

14. Johannsen DL, Tchoukalova Y, Tam CS et al (2014) Effect of 8 weeks of overfeeding on ectopic fat deposition and insulin sensitivity: testing the adipose tissue expandability hypothesis. Diabetes Care 37:2789-2797

15. Heilbronn LK, de Jonge L, Frisard MI et al (2006) Effect of 6month calorie restriction on biomarkers of longevity, metabolic adaptation, and oxidative stress in overweight individuals: a randomized controlled trial. JAMA 295:1539-1548

16. Bray GA, Smith SR, de Jonge L et al (2012) Effect of dietary protein content on weight gain, energy expenditure, and body composition during overeating: a randomized controlled trial. JAMA 307:47-55

17. Nguyen T, de Jonge L, Smith SR, Bray GA (2003) Chamber for indirect calorimetry with accurate measurement and time discrimination of metabolic plateaus of over $20 \mathrm{~min}$. Med Biol Eng Comput 41:572-578

18. Johannsen DL, Calabro MA, Stewart J, Franke W, Rood JC, Welk GJ (2010) Accuracy of armband monitors for measuring daily energy expenditure in healthy adults. Med Sci Sports Exerc 42:2134 2140

19. Bergstrom J (1975) Percutaneous needle biopsy of skeletal muscle in physiological and clinical research. Scand J Clin Lab Invest 35: 609-616

20. Obanda DN, Hernandez A, Ribnicky D et al (2012) Bioactives of Artemisia dracunculus L. mitigate the role of ceramides in attenuating insulin signaling in rat skeletal muscle cells. Diabetes 61:597605

21. Toledo FG, Watkins S, Kelley DE (2006) Changes induced by physical activity and weight loss in the morphology of intermyofibrillar mitochondria in obese men and women. J Clin Endocrinol Metab 91:3224-3227

22. Toledo FG, Menshikova EV, Ritov VB et al (2007) Effects of physical activity and weight loss on skeletal muscle mitochondria and relationship with glucose control in type 2 diabetes. Diabetes 56: 2142-2147

23. Pruchnic R, Katsiaras A, He J, Kelley DE, Winters C, Goodpaster BH (2004) Exercise training increases intramyocellular lipid and oxidative capacity in older adults. Am J Phys Endocrinol Metab 287:E857-E862

24. Bogacka I, Xie H, Bray GA, Smith SR (2005) Pioglitazone induces mitochondrial biogenesis in human subcutaneous adipose tissue in vivo. Diabetes 54:1392-1399

25. Lefort N, Glancy B, Bowen B et al (2010) Increased reactive oxygen species production and lower abundance of complex I subunits and carnitine palmitoyltransferase $1 \mathrm{~B}$ protein despite normal mitochondrial respiration in insulin-resistant human skeletal muscle. Diabetes 59:2444-2452 
26. Seifert EL, Estey C, Xuan JY, Harper ME (2010) Electron transport chain-dependent and -independent mechanisms of mitochondrial $\mathrm{H}_{2} \mathrm{O}_{2}$ emission during long-chain fatty acid oxidation. J Biol Chem 285:5748-5758

27. St-Pierre J, Buckingham JA, Roebuck SJ, Brand MD (2002) Topology of superoxide production from different sites in the mitochondrial electron transport chain. J Biol Chem 277:4478444790

28. Chappell JB, Perry SV (1954) Biochemical and osmotic properties of skeletal muscle mitochondria. Nature 173:1094-1095

29. Seifert EL, Bezaire V, Estey C, Harper ME (2008) Essential role for uncoupling protein-3 in mitochondrial adaptation to fasting but not in fatty acid oxidation or fatty acid anion export. J Biol Chem 283: 25124-25131

30. Amara CE, Marcinek DJ, Shankland EG, Schenkman KA, Arakaki LS, Conley KE (2008) Mitochondrial function in vivo: spectroscopy provides window on cellular energetics. Methods 46:312-318

31. Jubrias SA, Esselman PC, Price LB, Cress ME, Conley KE (2001) Large energetic adaptations of elderly muscle to resistance and endurance training. J Appl Physiol 90:1663-1670

32. Jubrias SA, Crowther GJ, Shankland EG, Gronka RK, Conley KE (2003) Acidosis inhibits oxidative phosphorylation in contracting human skeletal muscle in vivo. J Physiol 553:589-599

33. Conley KE, Jubrias SA, Esselman PC (2000) Oxidative capacity and ageing in human muscle. J Physiol 526:203-210

34. Blei ML, Conley KE, Kushmerick MJ (1993) Separate measures of ATP utilization and recovery in human skeletal muscle. J Physiol 465:203-222

35. Blei ML, Conley KE, Odderson IB, Esselman PC, Kushmerick MJ (1993) Individual variation in contractile cost and recovery in a human skeletal muscle. Proc Natl Acad Sci U S A 90:7396-7400

36. Kogot-Levin A, Saada A (2014) Ceramide and the mitochondrial respiratory chain. Biochimie 100:88-94
37. Yu J, Novgorodov SA, Chudakova D et al (2007) JNK3 signaling pathway activates ceramide synthase leading to mitochondrial dysfunction. J Biol Chem 282:25940-25949

38. Simoneau JA, Veerkamp JH, Turcotte LP, Kelley DE (1999) Markers of capacity to utilize fatty acids in human skeletal muscle: relation to insulin resistance and obesity and effects of weight loss. FASEB J 13:2051-2060

39. Toledo FG, Menshikova EV, Azuma K et al (2008) Mitochondrial capacity in skeletal muscle is not stimulated by weight loss despite increases in insulin action and decreases in intramyocellular lipid content. Diabetes 57:987-994

40. Coen PM, Menshikova EV, Distefano G et al (2015) Exercise and weight loss improve muscle mitochondrial respiration, lipid partitioning, and insulin sensitivity after gastric bypass surgery. Diabetes 64:3737-3750

41. Berggren JR, Boyle KE, Chapman WH, Houmard JA (2008) Skeletal muscle lipid oxidation and obesity: influence of weight loss and exercise. Am J Phys Endocrinol Metab 294:E726-E732

42. Samocha-Bonet D, Campbell LV, Mori TA et al (2012) Overfeeding reduces insulin sensitivity and increases oxidative stress, without altering markers of mitochondrial content and function in humans. PLoS One 7:e36320

43. Larsen S, Nielsen J, Hansen CN et al (2012) Biomarkers of mitochondrial content in skeletal muscle of healthy young human subjects. J Physiol 590:3349-3360

44. Dirks ML, Wall BT, van de Valk B et al (2016) One week of bed rest leads to substantial muscle atrophy and induces whole-body insulin resistance in the absence of skeletal muscle lipid accumulation. Diabetes 65:2862-2875

45. Gram M, Vigelsø A, Yokota T et al (2014) Two weeks of one-leg immobilization decreases skeletal muscle respiratory capacity equally in young and elderly men. Exp Gerontol 58:269-278 